\title{
Prenatal Androgens Defeminize Activation of GnRH Neurons in Response to Estradiol Stimulation
}

Ruth I. Wood*, Sung-Ji Kim*, and Douglas L. Foster†

*Department of Obstetrics \& Gynecology, Yale University School of Medicine, New Haven, CT 06520-8063, USA.

$\dagger$ TReproductive Sciences Program and Departments of Obstetrics \& Gynecology and Biology, University of Michigan, Ann Arbor, MI 48109-0404, USA.

Key words: Fos, immediate-early gene, LHRH, immunocytochemistry, sexual differentiation, sheep.

\begin{abstract}
To determine if prenatal androgens prevent activation of $\mathrm{GnRH}$ neurons in response to estradiol stimulation, Fos colocalization with GnRH was compared in the brains of normal female lambs, normal males, and androgenized females in response to a surge-inducing dose of estradiol. Blood samples were collected every $1-2 \mathrm{~h}$ for $6 \mathrm{~h}$ before estradiol treatment up to the time of sacrifice at $17-19 \mathrm{~h}$ posttreatment. Following perfusion, $60 \mu \mathrm{m}$ coronal brain sections were immunostained for Fos $(1: 1000$, Santa Cruz Biochemicals) and GnRH (1: 40,000, LR-1) using NiCl-enhanced and unenhanced DAB, respectively. Although LH secretion increased in females before sacrifice, no increase was observed in males or androgenized females. Despite differences in LH secretion, the number and distribution of GnRH neurons was not sexually dimorphic. Moreover, Fos immunostaining was visible throughout steroid-responsive limbic regions in all three groups of lambs. However, the colocalization of Fos with GnRH was highly sexually dimorphic. In females perfused after the peak of the LH surge, $65.7 \%$ of GnRH neurons in the preoptic area, anterior hypothalamus, and mediobasal hypothalamus expressed Fos, whereas only $1.7 \%$ of $\mathrm{GnRH}$ neurons were Fos-positive in males and androgenized females. These findings indicate that sex differences in the activation of $\mathrm{GnRH}$ neurons in response to estradiol are determined prenatally through the actions of testosterone.
\end{abstract}

In sheep, as in many species, the surge mode of gonadotropin secretion is functional only in the female. Adult males seldom produce a surge of $\mathrm{LH}$, even when stimulated with exogenous estradiol (1). Moreover, exogenous estradiol induces an LH surge in the developing female lamb, but only rarely in the male or androgenized female (2). In the female sheep, the LH surge is accompanied by a massive release of GnRH into the hypophysealportal vasculature, which provides the key stimulus for $\mathrm{LH}$ release (3). The absence of a preovulatory LH surge in the male sheep reflects the actions of steroids prenatally to prevent estradiolstimulated GnRH release, as GnRH-stimulated LH release is not sexually differentiated in male and female sheep (4). Our companion manuscript provides direct evidence that estradiol stimulation reliably induces a surge of $\mathrm{GnRH}$ in female lambs, but only occasionally in males or androgenized females (5).

Having determined that GnRH is not released in males in response to estradiol stimulation, a next logical question is to determine if GnRH neurons in the male and androgenized female sheep are activated in response to the estrogen stimulus, or if they remain quiescent at the time that the female generates a preovulatory surge. This is critical to our understanding of whether GnRH neurons in the male are stimulated, but fail to release substantial quantities of GnRH into the portal capillaries, or whether prenatal androgens block activation of GnRH neurons in response to estradiol stimulation. To address this question, we used the expression of c-fos, one of the family of immediate-early genes, as a marker of activation of GnRH neurons. Using immunocytochemistry for Fos, the protein-product of the c-fos gene, in combination with GnRH immunohistochemistry, Moenter et al. (6) demonstrated that a majority of GnRH neurons in the adult ewe express Fos during an estradiol-induced GnRH surge. Moreover, there is precedent for sex differences in expression of Fos in GnRH neurons. In the ferret, which ovulates only in response to mating, Lambert et al. (7) reported that GnRH neurons contain Fos after mating in female ferrets, but not in males. In rats, estradiol-progesterone stimulation induced Fos in GnRH neurons in females, but not in males (8). The present study has extended these findings, comparing Fos activation of GnRH neurons in normal males, normal females, and androgenized females to test the hypothesis that prenatal androgens prevent activation of GnRH neurons in response to estradiol stimulation.

\section{RESULTS}

\section{$L H$ secretion in response to estradiol stimulation}

Fig. 1 illustrates LH concentrations in blood samples collected from males, females, and androgenized females beginning $6 \mathrm{~h}$

Correspondence to: Ruth. I Wood, Department of Obstetrics and Gynecology, Yale University School of Medicine, 333 Cedar Street, Box 208063, New Haven, CT 06520-8063, USA.

(C) 1996 Blackwell Science Ltd 
before estradiol treatment and continuing up to the time of perfusion (17-19 h post-estradiol). Before estradiol treatment, LH concentrations averaged $12.6 \pm 0.7 \mathrm{ng} / \mathrm{ml}$ in all three groups of lambs and reached a nadir of $3.0 \pm 0.4 \mathrm{ng} / \mathrm{ml} 8 \mathrm{~h}$ after insertion of estradiol capsules. In males and androgenized females, $\mathrm{LH}$ concentrations in serum remained low until sacrifice $10-12 \mathrm{~h}$ later. In 4 of 5 female lambs, estradiol stimulation induced a surge of LH which peaked $17 \mathrm{~h}$ after the beginning of estradiol. In the one remaining female, LH secretion increased before sacrifice, but failed to achieve a high concentration in circulation.

\section{GnRH immunoreactivity in the postpubertal sheep brain}

Fig. 2 presents photomicrographs of GnRH-immunoreactive neurons with (Fig. 1A, D) or without (Fig. 1B) Fos immunoreactivity. Fig. 3 illustrates single and double-labelled GnRH neurons in the diagonal band of Broca (DBB), medial preoptic area (MPOA), anterior hypothalamus (AH), and mediobasal hypothalamus $(\mathrm{MBH})$ of a representative female lamb during an estrogeninduced surge of LH. Fig. 4 depicts the mean \pm SEM number of GnRH neurons per section in males, females, and androgenized females from the tenia tecta rostrally to the mammillary bodies, and the percentage of those neurons with nuclear Fos immunoreactivity. The distribution of GnRH-immunoreactive neurons in the postpubertal lamb was similar to that described previously for the mid-gestation ovine fetus (9) and for the mature ewe (10). The majority of GnRH-immunoreactive neurons were found in the preoptic area, with additional stained cells scattered rostrally in the diagonal band of Broca and tenia tecta, and sparsely distributed caudally throughout the anterior and mediobasal hypothalamus. The number and distribution of GnRH neurons was not different in males, females, and androgenized females $(\mathrm{P}>0.05)$.

\section{Fos immunoreactivity and colocalization with GnRH}

In male, female, and androgenized female lambs, Fosimmunoreactive nuclei were abundant and widely distributed in the preoptic area and hypothalamus after estradiol stimulation. In all lambs, nuclei immunostained for Fos were present in the same regions that contain GnRH neurons. However, colocalization of Fos with GnRH was highly sexually dimorphic $(\mathrm{P}<0.05)$. In the four females perfused after the peak of $\mathrm{LH}$ release, Fos was present in $65.7 \%$ of GnRH neurons in the preoptic area, anterior hypothalamus, and mediobasal hypothalamus. There was no correlation between the number of GnRH neurons and the extent of Fos activation in these areas. Rostrally, in the diagonal band of Broca of females, few GnRH neurons expressed Fos (3.1\%). For the single female perfused before the peak of $\mathrm{LH}$ release, Fos was visible in only 3 of $173 \mathrm{GnRH}$ neurons examined $(1.7 \%)$. Despite the low incidence of Fos expression in this female, her data were included in statistical analyses. In males and androgenized female lambs, few GnRH neurons expressed Fos. Of the $3039 \mathrm{GnRH}$ neurons examined from 11 brains (5 androgenized females, 6 males), Fos was present in only 51 neurons (1.7\%). No GnRH neurons in DBB of males and androgenized females expressed Fos. In other areas, Fos-positive GnRH neurons were scattered within MPOA, AH, and MBH.

\section{Discussion}

The present study demonstrates that sexual differentiation of the neuroendocrine response to estradiol stimulation is determined, at least in part, through sex differences in the activation of GnRH neurons as determined by the pattern of Fos expression. Although approximately two-thirds of GnRH neurons in the female lamb brain expressed Fos in response to a surge-inducing dose of estradiol, Fos was not evident in substantial numbers of $\mathrm{GnRH}$ neurons from males or androgenized females. This sex difference in Fos expression within GnRH neurons suggests that prenatal testosterone from the fetal testes prevents the postnatal activation of the GnRH neurons in response to estradiol.

The capacity to produce a preovulatory surge of LH in response to estrogen is sexually differentiated in most species, except primates (reviewed in 11). Although estradiol induces a massive release of LH from the pituitary in female sheep, in males the LH surge is neither present nor readily inducible (1). It is important to note that in the sheep, progesterone is not required for expression of the preovulatory LH surge (12), and treatment of female lambs with progesterone will block the facilitatory effects of estradiol (13). In sheep, and presumably in other species, the preovulatory surge of LH is stimulated by a surge of GnRH secretion (3). The ability to monitor GnRH in the hypophyseal-portal vasculature has recently permitted us to directly test the hypothesis that sexual differentiation of the $\mathrm{LH}$ surge is due to sex differences in estradiol-stimulated GnRH release. The results of that study, presented in the preceding companion paper, indicate that the pattern of estradiol-stimulated $\mathrm{GnRH}$ release is sexually dimorphic (5). Moreover, through the use of Fos to identify GnRH neurons activated by estradiol stimulation, the present study reveals that sex differences in the secretion of GnRH reflect sex differences in activation of $\mathrm{GnRH}$ neurons. These sex differences arise as a result of testosterone exposure during prenatal development.

Previous studies in several species, including sheep, have revealed that the number and distribution of $\mathrm{GnRH}$ neurons is not sexually dimorphic $(9,14,15)$. The absence of sex differences in GnRH neuroanatomy in the face of substantial sex differences in control of GnRH secretion implies that the expression of the preovulatory GnRH surge is not localized to a sex-specific population of GnRH neurons. The induction of Fos immunoreactivity in GnRH neurons after estradiol stimulation supports and extends this conclusion. In particular, the even distribution of Fos in GnRH neurons of female lambs throughout the preoptic area, anterior hypothalamus, and mediobasal hypothalamus, and the almost complete absence of Fos in GnRH neurons of males and androgenized females in these same brain regions, provides no evidence of a surge-specific population of GnRH neurons.

Fos activation of GnRH neurons in response to estradiol stimulation has been previously examined in female sheep (6), rats (8), and primates (16). In sheep, Moenter et al. (6) reported findings similar to those of females in the present study. In particular, Fos was not present in substantial numbers of $\mathrm{GnRH}$ neurons until after the peak of the LH surge, and even in those ewes with substantial Fos/GnRH colocalization, few GnRH neurons rostrally in DBB expressed Fos. In rats, although Fos activation of GnRH neurons was not correlated with $\mathrm{LH}$ values, the findings were qualitatively similar (8). As in the sheep, Fos activation was notably absent in GnRH neurons rostral to the preoptic area. Based on the peculiar developmental migration of the GnRH neurons $(17,18)$, it is tempting to speculate that the 'silent' GnRH neurons in rostral forebrain do not participate in $\mathrm{GnRH}$ release at the median eminence as a result of either 
A
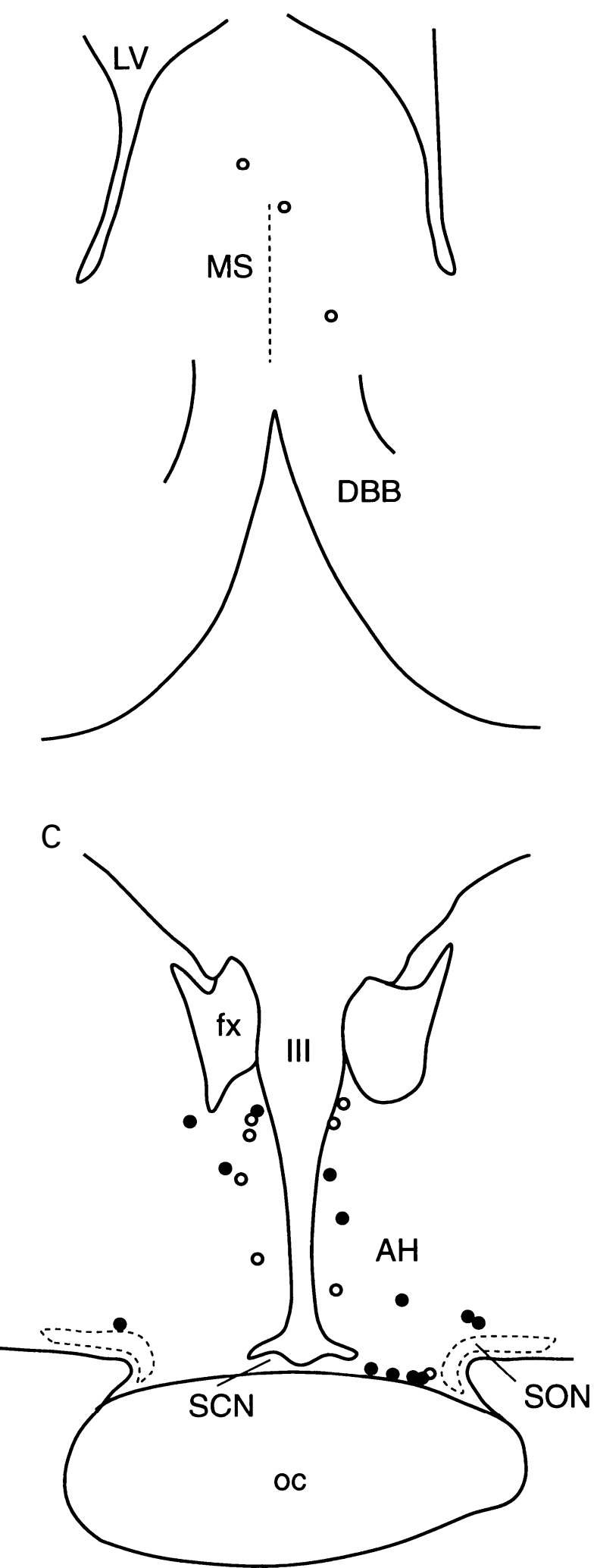

B

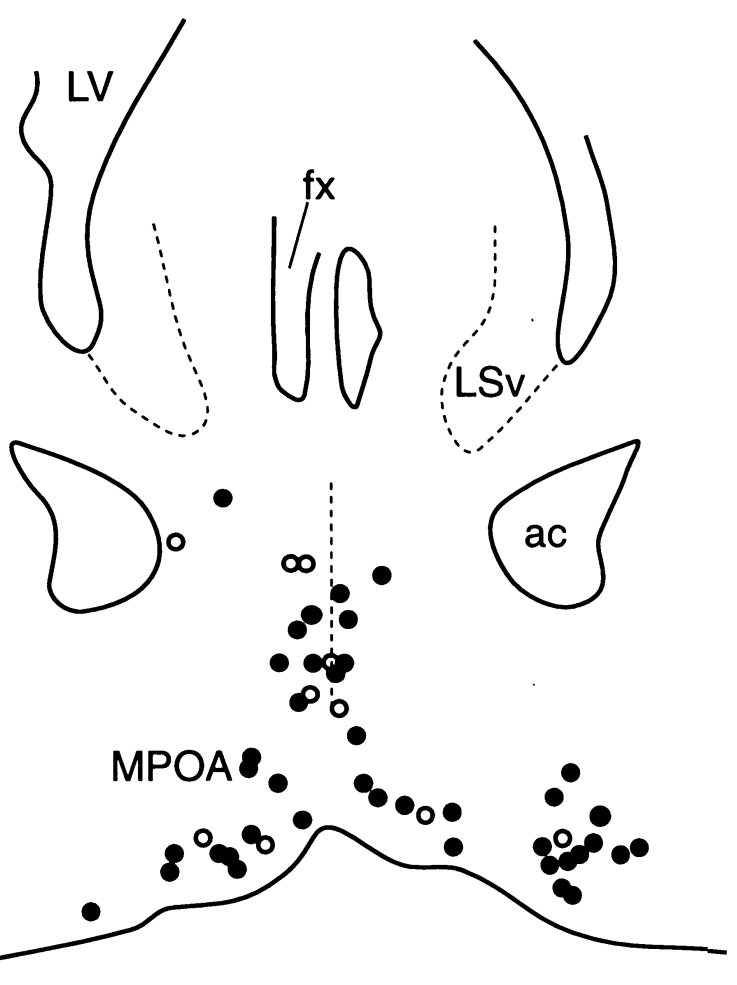

D

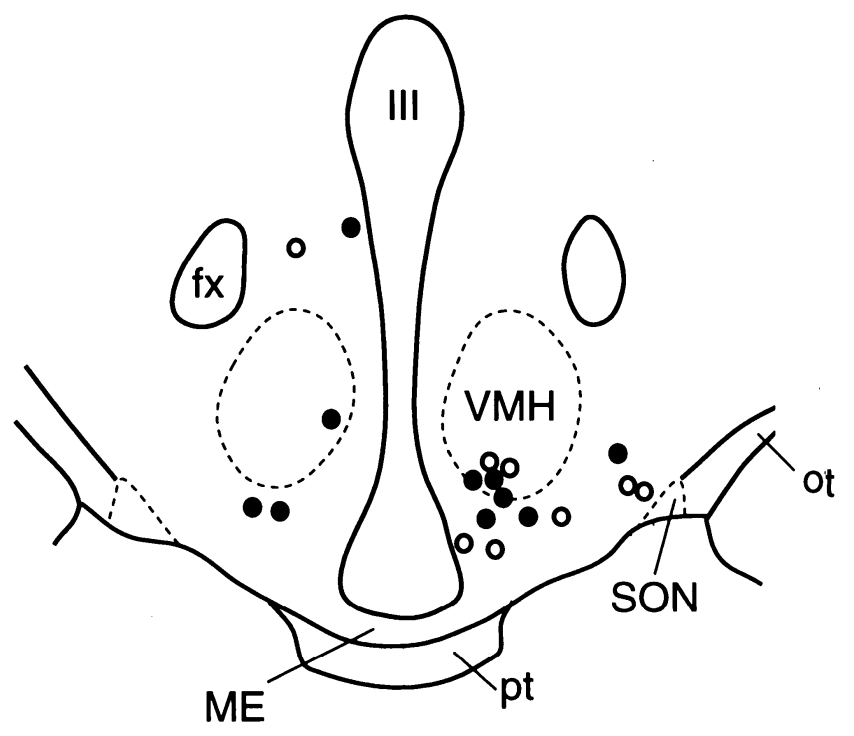

FIG. 3. The distribution of GnRH neurons in a female lamb brain in the presence (solid circles) and absence (open circles) of nuclear Fos immunoreactivity in the diagonal band of Broca (A), preoptic area (B), anterior hypothalamus (C), and mediobasal hypothalamus (D). Abbreviations: III: third ventricle, ac: anterior commissure, AH: anterior hypothalamus, DBB: diagonal band of Broca, fx: fornix, LSv: ventral lateral septum, LV: lateral ventricle, ME: median eminence, MPOA: medial preoptic area, MS: medial septum, oc: optic chiasm, ot: optic tract, pt: pituitary, SCN: suprachiasmatic nucleus, SON: supraoptic nucleus, VMH: ventromedial nucleus of the hypothalamus. 
arrested or delayed migration from the olfactory placode during prenatal development. In the female primate, Fos is not detected in GnRH neurons during the preovulatory surge (16), which may indicate that $\mathrm{GnRH}$ release in this species is controlled at $\mathrm{GnRH}$ neuron terminals in the median eminence.

We must consider the possibility that GnRH neurons of males and androgenized females might express Fos in greater numbers if perfusions were delayed. As described in the preceeding paper, any increases in estradiol-stimulated GnRH secretion from males and androgenized females are both delayed and attenuated relative to those in normal females (5). This same phenomenon has been observed in other studies measuring estradiol-induced LH secretion $(19,20)$. However, neuroendocrine responses to estradiol stimulation in males and androgenized females are inconsistent both within and among individuals, in terms of expression, timing, and magnitude $(19,20)$. Thus, in the present study, all lambs were perfused at the same time after estradiol treatment to facilitate comparisons among groups of lambs. Moreover, even if it were possible to perfuse all lambs shortly after the peak of GnRH release for each individual, the incidence of Fos colocalization with GnRH would likely be less in males and androgenized females, based on the low amplitude of GnRH release in these lambs (5).

It is reasonable to expect that sexual differentiation of the GnRH neurosecretory system is primarily through the actions of testosterone on steroid receptor-containing neurons, and not on GnRH neurons directly. Studies in several species have shown that GnRH neurons do not contain androgen or estrogen receptors (21-24). Instead, prenatal testosterone acting on steroid-responsive neurons which project to $\mathrm{GnRH}$ neurons may determine sex differences in synaptic input to GnRH neurons, as has been reported for the rat (25). The increased innervation of GnRH neurons in females could be responsible, at least in part, for sex differences in the responsiveness of GnRH neurons to estradiol.

Two previous studies have examined sex differences in Fos activation of GnRH neurons. In ferrets, mating stimulates LH secretion only in females, and Fos expression in GnRH neurons was observed only in brains of mated females (7). Likewise, in rats, Fos was not detected in GnRH neurons from males when stimulated with estradiol and progesterone (8). The present study extends these findings to include the examination of possible mechanisms involved in such sex differences. The lack of Fos expression in GnRH neurons from androgenized females suggests that prenatal testosterone exposure prevents postnatal estradiolinduced activation of GnRH neurons. As the present study used testosterone-treated females, it was not possible to determine if the observed effects are mediated by conversion to estradiol. However, based on studies of defeminization of the LH surge in the rat (26), it is probable that prenatal estradiol derived from aromatization of testosterone defeminizes the GnRH neurosecretory system in the lamb.

There do exist certain caveats for using Fos to identify neurons that are activated in response to a particular treatment. First, the absence of Fos immunostaining in GnRH neurons from males and androgenized females does not indicate that these neurons are 'inactive'. Instead, we presume that $\mathrm{GnRH}$ neurons in males and androgenized females are capable of normal tonic secretion, as has been demonstrated in the adult ram (27). Fos is not detectable in GnRH neurons from female sheep in the absence of steroid feedback, when GnRH pulsatility is high (6). Therefore, it appears that Fos is expressed only in response to acute stimuli, but the protein is not a good indicator for chronic neural activity. Secondly, there exists the possibility that other immediate-early genes besides Fos may be expressed in response to estradiol stimulation of males and androgenized females. However, such a finding would not detract from the observation of sex differences in Fos reported herein. Finally, it is not possible to determine from the present study if Fos activation drives GnRH release during the surge, or is expressed as a consequence of increased GnRH neurosecretion (6). The lack of Fos expression in GnRH neurons from the single female perfused before the peak of $\mathrm{GnRH}$ release, and from a similar female sheep in the study of Moenter et al. (6), argues for the latter interpretation.

Interpretation of underlying biochemical processes related to sex differences which involve Fos expression is difficult because the cellular actions of Fos on GnRH neurons remain unclear. It may be that Fos is a measure of stimulation received by the neuron. Alternatively, Fos may be a signal for replenishment of GnRH stores following release. Despite these caveats, the sex differences in Fos activation noted in the present study suggest that males and females have different neural responses to estradiol stimulation, which result in a sex difference in GnRH secretion, and that these differences are governed by prenatal exposure to gonadal steroids.

\section{Materials and methods}

\section{Animals}

Male, female, and androgenized female lambs ( $\mathrm{n}=5$ each) were purchased in the previous year from Wolf Creek Farms (Hubbard Lake, MI, USA), and transported to the Reproductive Sciences Program Sheep Research Facility in Ann Arbor at one week of age. Androgenized female lambs were masculinized by exposure to testosterone cypionate as described in the preceding paper (weekly maternal injections of $200 \mathrm{mg}$ from 30 to 86 days of gestation, ref. 5). This androgen treatment heavily masculinized both the external genitalia and the control of reproductive neuroendocrine function (28). Male and female lambs that received no prenatal steroids served as controls.

\section{Gonadectomy and steroid replacement}

Postnatally, lambs were treated as in the preceding paper (5). Specifically, all lambs were gonadectomized and steroids were replaced by means of a Silastic capsule containing estradiol. As determined from the pattern of LH secretion in blood samples collected twice each week, all lambs were neuroendocrinologically mature at the time of sacrifice in February at 45 weeks of age (28). In the estradiol-treated gonadectomized lamb, a sustained rise in circulating LH concentrations from such samples collected infrequently reflects an increase in LH pulse frequency resulting from the pubertal reduction in sensitivity to inhibitory steroid feedback (29, for review).

\section{Surge LH secretion}

We used the same surge induction protocol as for our previous experiments characterizing hormone release $(5,19)$. Specifically, to maximize the amplitude of estradiol-stimulated $\mathrm{GnRH} / \mathrm{LH}$ release, the study was conducted 3 weeks after removal of estradiol implants which had provided chronic inhibitory steroid feedback since 2 weeks of age. Three weeks of steroid deprivation is sufficient for lambs to acheive hourly LH pulses (2), comparable to that in post-pubertal lambs (30). Surges induced in ovariectomized females are comparable to those in females with chronic estradiol treatment (12). At the time of surge induction, the pattern of gonadotropin release was evaluated by measuring $\mathrm{LH}$ in blood samples collected every $2 \mathrm{~h}$ beginning $4 \mathrm{~h}$ before, and continuing for $18 \mathrm{~h}$ after implantation of four 30-mm Silastic estradiol capsules. Additional samples were collected hourly beginning $12 \mathrm{~h}$ after estradiol stimulation. Lambs 
ence for an embryonic origin in the olfactory placode. Brain Res Dev Brain Res 1989; 46: 309-318.

18 Schwanzel-Fukuda M, Pfaff DW. Origin of luteinizing hormonereleasing hormone neurons. Nature 1989; 338: 161-164.

19 Wood RI, Foster DL. Prenatal androgens and the timing of seasonal reproductive transitions in sheep. Biol Reprod 1992; 47: 389-396.

20 Wood RI, Mehta V, Herbosa CG, Foster DL. Prenatal testosterone differentially masculinizes tonic and surge modes of luteinizing hormone secretion in the developing sheep. Neuroendocrinology 1995; 62(3): $215-318$.

21 Lehman MN, Karsch FJ. Do gonadotropin-releasing hormone, tyrosine hydroxylase-, and beta-endorphin-immunoreactive neurons contain estrogen receptors? A double-label immunocytochemical study in the Suffolk ewe. Endocrinology 1993; 133: 887-895.

22 Herbison AE, Robinson JE, Skinner DC. Distribution of estrogen receptor-immunoreactive cells in the preoptic area of the ewe: co-localization with glutamic acid decarboxylase but not luteinizing hormone-releasing hormone. Neuroendocrinology 1993; 57: 751-759.

23 Herbison AE, Theodosis DT. Localization of oestrogen receptors in preoptic neurons containing neurotensin but not tyrosine hydroxylase, cholecystokinin or luteinizing hormone-releasing hormone in the male and female rat. Neuroscience 1992; 50: 283-298.

24 Shivers BD, Harlan RE, Morrell JI, Pfaff DW. Absence of oestradiol concentration in cell nuclei of LHRH-immunoreactive neurones. Nature 1983; 304: 345-347.

25 Chen W-P, Witkin JW, Silverman AJ. Sexual dimorphism in the synaptic input to gonadotropin releasing hormone neurons. Endocrinology 1990; 126: 695-702.

26 Korenbrot CC, Paup D, Gorski RA. Effects of testosterone or dihydrotestosterone propionate on plasma FSH and LH levels in neonatal rats and on sexual differentiation of the brain. Endocrinology 1995; 97: 709-717.
27 Jackson GL, Kuehl D, Rhim TJ. Testosterone inhibits gonadotropinreleasing hormone pulse frequency in the male sheep. Biol Reprod 1991; 45: 188-94.

28 Herbosa CG, Wood RI, Foster DL. Prenatal androgens modify the reproductive response to photoperiod in the developing sheep. Biol Reprod 1995; 52: 1-7.

29 Foster DL. Puberty in the female sheep. In: Knobil E \& Neill JD (eds) The Physiology of Reproduction, New York: Raven Press, 1994: 411-451.

30 Karsch FJ, Dierschke DJ, Weick RF et al. Positive and negative feedback control by estrogen of luteinizing hormone secretion in the rhesus monkey. Endocrinology 1993; 92: 799-804

31 Foster DL. Preovulatory gonadotropin surge system of prepubertal female sheep is exquisitely sensitive to the stimulatory feedback action of estradiol. Endocrinology 1984; 115: 1186-1189

32 Winans SS, Powers JB. Olfactory and vomeronasal deafferentation of male hamsters: histological and behavioral analyses. Brain Res 1977; 126: 325-344.

33 Hauger RL Karsch FJ, Foster DL. A new concept for control of the estrous cycle of the ewe based upon temporal relationship between luteinizing hormone, estradiol, and progesterone in peripheral serum and evidence that progesterone inhibits tonic $\mathrm{LH}$ secretion. Endocrinology 1977; 101: 807-817.

34 Ebling FJP, Schwartz ML, Foster DL. Endogenous opioid regulation of pulsatile luteinizing hormone secretion during sexual maturation in the female sheep. Endocrinology 1989; 125: 369-383.

35 Niswender GD, Reichert $\mathrm{Jr}$ LE, Midgley AR, et al. Radioimmunoassay for bovine and ovine luteinizing hormone. Endocrinology 1969; 84: 1166-1173. 